\title{
Introduction to Part 2 of the Special Issue on Ground Improvement Techniques
}

\author{
M. I. M. Pinto*, I. Jefferson ${ }^{\dagger}$ and A. S. Cardoso* \\ ${ }^{*}$ FCTUC, Universidade de Coimbra, Portugal; ${ }^{\dagger}$ School of Engineering, University of \\ Birmingham, UK; ${ }^{\star}$ Faculdade de Engenharia, Universidade de Porto, Portugal
}

This issue comprises a selection of papers that were initially presented at the sixth International Conference on Ground Improvement Techniques, held in the University of Coimbra, Portugal, in July 2005. A total of eleven selected papers are published as a special issue in two parts. Part 1 was published as Volume 11, Number 1, January 2007, with five of the selected papers. These papers covered a number of themes, including cement treatment of soils, use of geosynthetics for soil reinforcement and soil densification to mitigate earth-quake induced liquefaction. The final six papers are published as Part 2, herein. The six papers presented focus on deep improvement techniques from procedures and implementation, to performance and quality assessment. Also included in these papers are presentations of case histories together with results from laboratory, field and numerical studies. A brief summary of each paper is given to the readers in this editorial.

Lee and Karunaratne examined pre-consolidation of soft soils accelerated by the use of pre-fabricated vertical drains manufactured from a biodegradable jute and coir fibres, which offer a potentially more sustainable vertical drain material. Applications to a number of projects in south-east Asia have been presented, highlighting the advantages to be gained by this approach.

Gopal Madabhushi's paper deals with the use of a dynamic centrifuge modelling as a tool to investigate earthquake-induced liquefaction mechanisms. Three ground improvement methods: densification, drainage and grouting, were considered. From this, the ability of dynamic centrifuge modelling to monitor the performance of the improved ground in mitigating the liquefaction risk has been highlighted.
The influence of possible defects is a very important issue in relation to tunnelling works and this has been studied by Flora et al., who focused on jet grouting columns for a temporary support of tunnelling. A design criterion was developed and proposed based on a statistical analysis of some field data collected and a probabilistic approach by using a numerical simulation. From this, Costabile et al. produced a simple way to plan the number, spacing and diameter of jet grouted tunnel support umbrellas.

Serridge and Synac share recent experience on ground treatment of soft soils for a recent motorway widening and new highway construction. The different stages of construction, from site investigation to ground improvement design, implementation and finally monitoring and quality control are addressed in a very practical perspective.

The rehabilitation of railway lines and the need of improving the ground beneath the existing rail tracks without interference of rail elements has been assessed by Adam et al. They examined the application of different deep improvement techniques, including deep vibrations, deep mixing and column construction, and in addition presented a case history for illustration. This paper further describes the use of a fully mechanised ballast excavation and recycling machine for the surface-near formation rehabilitation.

In another paper, Adam et al. propose and describe an integrated dynamic compaction control method for heavy tamping (deep dynamic compaction). This method is based on measurements of the acceleration of the falling weight and of the decay of the amplitude of the free vibrations of the soil after each impact. By this it is possible to perform a continuous quality control of compaction enabling the optimisation of the compaction procedure to take place. 